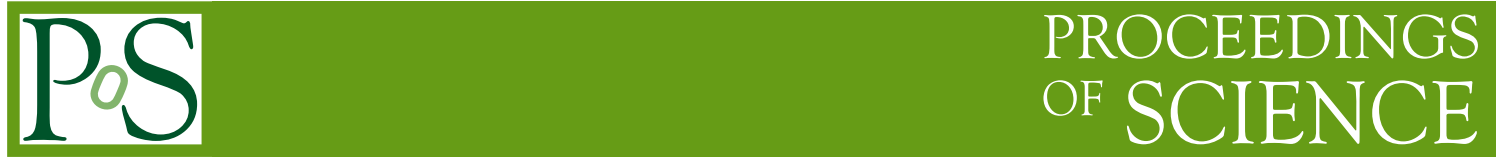

\title{
Are the two peaks of the Cathedral QPO real harmonics?
}

\section{Jerome Rodriguez*}

CEA Service dÁstrophysique, Laboratoire AIM, Saclay, France

E-mail: jrodriguez@cea.fr

\section{Peggy Varnière}

CNRS, Laboratoire APC, Paris, France

\begin{abstract}
We present a study of the two main peak of the so-called cathedral QPO in XTE J1859+226. While looking at the temporal evolution of the two features we show that they do not manifest the same amplitude of variations of their power, and do not seem to follow the flux variations in the same way. We then present their RMS-spectra and show that they do not have the same shape, slope and cut-off energy. We discuss these different facts and try to answer the question regarding the genuineness of their harmonic relationship
\end{abstract}

Fast $X$-ray timing and spectroscopy at extreme count rates

February 7-11, 2011

Champéry, Switzerland

\footnotetext{
${ }^{*}$ Speaker.
} 


\section{Introduction}

One of the very actual question regarding the physics at work in black hole binaries (BHB, a.k.a microquasars e.g. Remillard \& McClintock 2006, for a review) concerns the orgin and nature of the quasi periodic oscillations (QPO) one can see while producing the power density spectra (PDS) of these sources. While low frequency QPOs (LFQPO) have been commonly seen in almost all BHBs in their hardest states Remillard \& McClintock (2006); Homan \& Belloni (2005), high frequency QPOs have only been seen in a handful of them. In the study presented here we focus on LFQPOs. The latter have been further classified into types A, B, or C based on their typical frequencies, total RMS amplitude, time lags, and the overal shape of underlying continuum of the PDS (e.g. Remillard et al. 2002; Casella et al. 2005). We have recently proposed a tentative classification of states based on the presence of the different types of QPOs (Varnière et al. 2011).

Many models have been proposed to explain the origin and behaviour of these LFQPO, but none of them has thus far been able to explain all observational facts. It is, indeed, quite clear that the inner disk somehow sets the frequency of LFQPOs (e.g. Muno et al. 1999; Rodriguez et al. 2002a,b, 2004b; Mikles et al. 2009), but LFQPOs have high amplitudes in states dominated by emission at hard X-rays, and recent studies have shown that their frequency is (also) correlated with the power law photon index (e.g. Vignarca et al. 2003; Shaposhnikov \& Titarchuk 2007). This could indicate a strong relation to the corona. Finally the RMS-spectra of LFQPO is hard (it increases with the energy) but also presents a cut-off whose energy is variable (Rodriguez et al. 2004a, 2008).

A much less explored properties of these features is related to the presence, and behaviour of (sub-) harmonically related peaks in the PDS. These harmonic can, in some cases, have properties that differ significantly from those of the fundamental QPO. This is, for example, the case of the type B QPO. For the latter, the fundamental and harmonics show opposite signs of their times lags, and also different shape of their RMS-spectra (Casella et al. 2004; Cui 1999; Homan et al. 2001; Rao et al. 2010). In this paper we study the particular case of the so-called "Cathedral" QPO seen in the microquasars XTE J1859+226 Casella et al. (2004). This letter is a summary of our recently accepted paper (Rodriguez \& Varnière 2011). It is organised as follows: in the next section we give a brief introduction on XTE J1859+226 and its temporal properties. We first present the basic properties of the peaks (Sec. 3), and then present their temporal evolution (Sec. 4) and spectra (Sec. 5). In regards of these analysis we discuss the potential association of the two peaks in the last section.

\section{XTE J1859+226}

XTE J1859+226 was discovered on 1999 October 9 with the RXTE All Sky Monitor (Wood et al. 1999) as it was entering into outburst. It is a microquasar given the observations of relativistic ejections in radio (Brocksopp et al. 2002). The RXTE/ASM and GBI $2.25 \mathrm{GHz}$ light curves of the source are represented in Fig. 1. Cui et al. (2000) observed LF and HFQPOs which led them to classify XTE J1859+226 as a candidate BHB. An extensive timing analysis of this source is presented by Casella et al. (2004).XTE J1859+226 displays all three types of LFQPOs, and, in two particular observations (on MJDs 51474.43 and 51475.43, Fig. 1), Casella et al. (2004) observed 


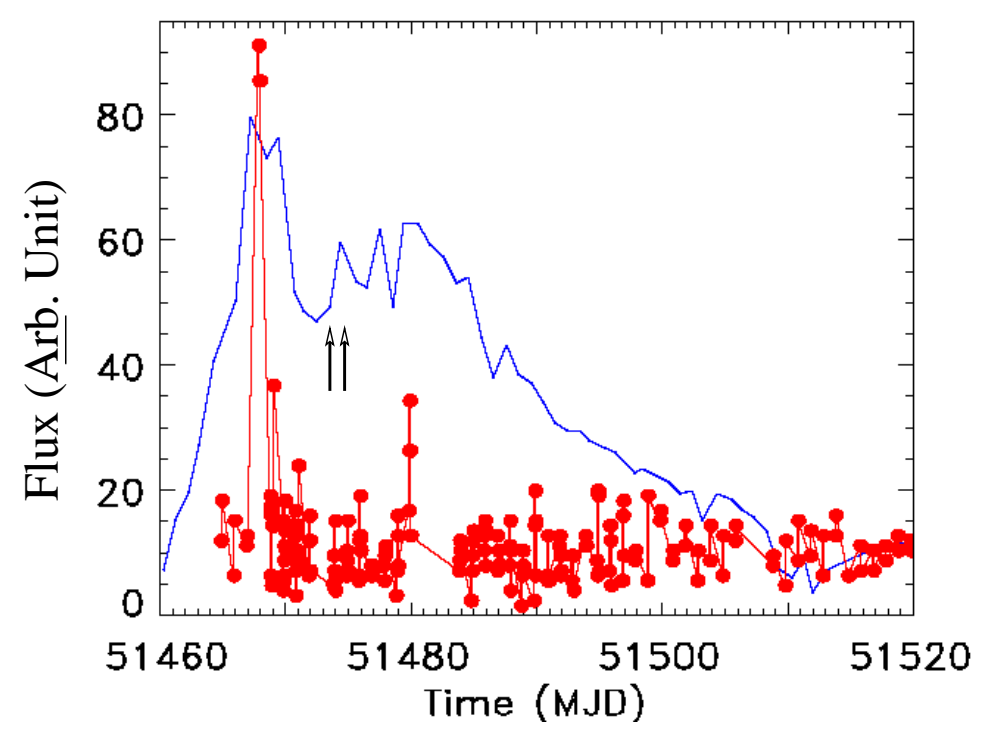

Figure 1: RXTE/ASM (1.2-12 keV, blue) and Green Bank Interferometer (2.25 GHz, red) light curves of the 1999 outburst of XTE J1859+226. The two vertical arrows mark the position of the RXTE observations analysed in this paper.

the presence of two peaks with harmonically related frequencies, but unlike any other cases, similar RMS-amplitudes, that they dubbed 'Cathedral'-QPO. Interestingly these authors remarked that the strongest peak (and highest in frequency, hereafter Peak 2) has hard lags (the hard X-ray lag behind the soft X-rays), while the lowest frequency peak (Peak 1) has soft lags. In this respect Peak 2 was considered as the fundamental, while Peak 1 was the sub-harmonic.

\section{Broad band fitting of the PDSs}

The broad band PDSs of both observations were fitted with the additions of several Lorentzians (see Rodriguez \& Varnière 2011, for the details of the data reduction and procedures of fittings). We focus in the remaining of the two thin features at $\sim 3$ and $\sim 6 \mathrm{~Hz}$. Fig. 2 shows the example of the fit to the PDS of the second observation (MJD 51475.43). In both observation Peak 2 may have a complex structure, and in fact it seems to be better represented by 2 thin Lorentzians (Fig. 2 ). Note that Casella et al. (2004) also make a similar remark, but only in the case of the second observation. This additional feature is, however, poorly defined, and its parameters are badly constrained. We verify, by re-doing the whole analysis that it had no significant impact on the other peaks, and since no influence was found it was omitted from our study, and is not further discussed here.

The best peak parameters are the following for Obs. 1 (resp. Obs. 2) $v_{1}=2.94 \mathrm{~Hz}, Q_{1}=5.9$, $A_{1}=2.8 \%\left(\right.$ resp. $v_{1}=3.00 \mathrm{~Hz}, Q_{1}=5.2, A_{1}=2.9 \%$ ), and $v_{2}=5.83 \mathrm{~Hz}, Q_{2}=7.3, A_{2}=4.7 \%$ (resp. $v_{2}=5.86 \mathrm{~Hz}, Q_{2}=6.5, A_{2}=4.6 \%$ ).

\section{Temporal evolution}

We produced the dynamical PDS of XTE J1859+226 to look to any temporal evolution of the main peaks during each of the observations (Fig. 3). Both observation show similar temporal 


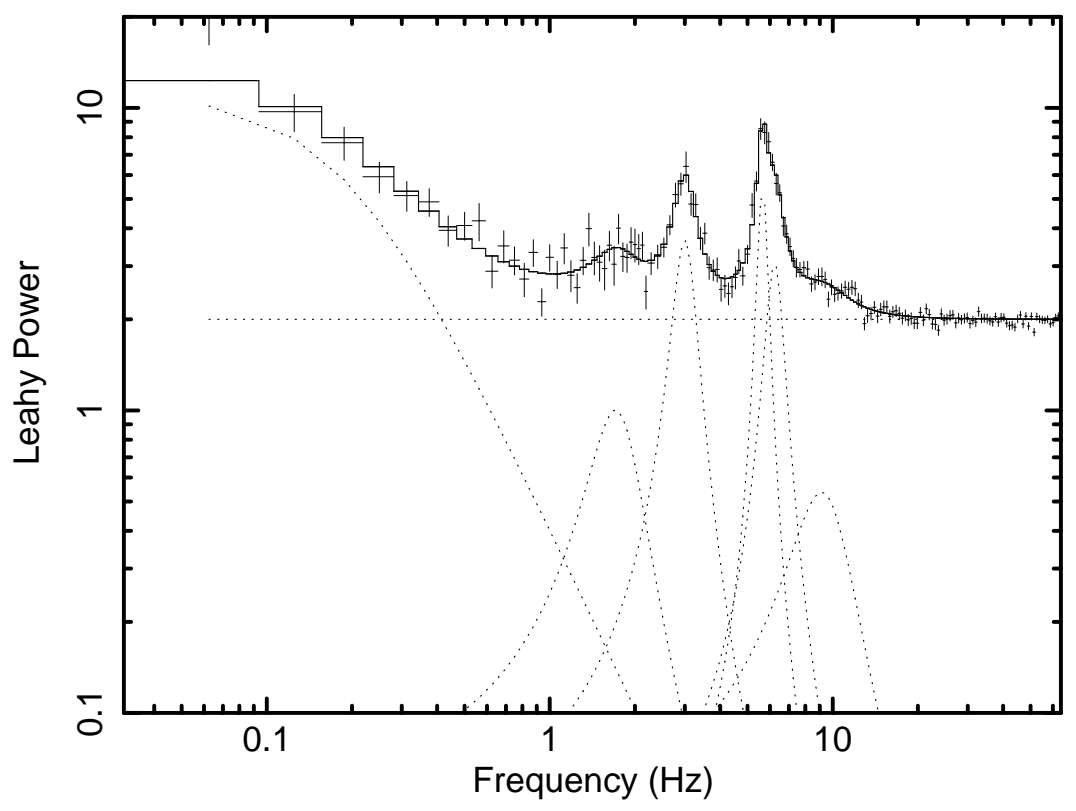

Figure 2: Broad band fit of the PDS of the second observation. The dashed lines represent the different individual components.

dependences of the two peaks. In both cases Peak 1 seems, on average, much weaker than Peak 2. It is strong only when the count rate is around its mean value. It is, in particular quite weak during the small flares, and is not visible during the dips. Peak 2, on the other hand, seems, in term of power, more stable and seems to vary significantly only during the dips, where it may disappear. In fact a

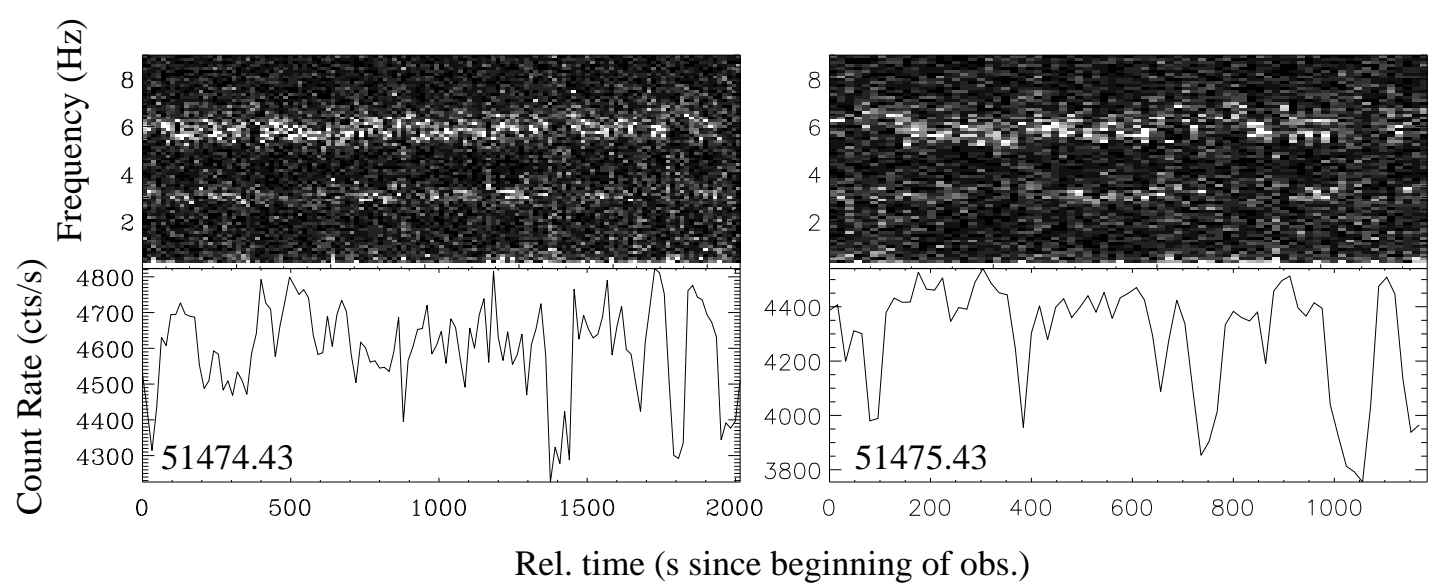

Figure 3: Dynamical PDS (top panels) and RXTE/PCA light curves of XTE J1859+226 during the two observations. Left: Obs. 1. Right: Obs.2

proper study of the dependences of the properties of the two peaks with the count rate (Rodriguez \& Varnière 2011) indeed shows that both features undergo very different evolution with the count rate. The amplitude of Peak 1 decreases significantly with increasing count rate, while, at the same time, the amplitude of Peak 2 may show a linear increase. 


\section{Energy dependences}

The energy dependences of the peak's RMSs (in other words the RMS-Spectra) are reported in Fig. 4 for Peaks 1, 2, and the additional thin Lorentzian added to better represent Peak 2 (Sec. 3). Note, that, as mentioned in Sec. 3, the shapes of the RMS-Spectra of Peak 1 and 2 are the same if the third Lorentzian is not considered in the fits (Rodriguez \& Varnière 2011).

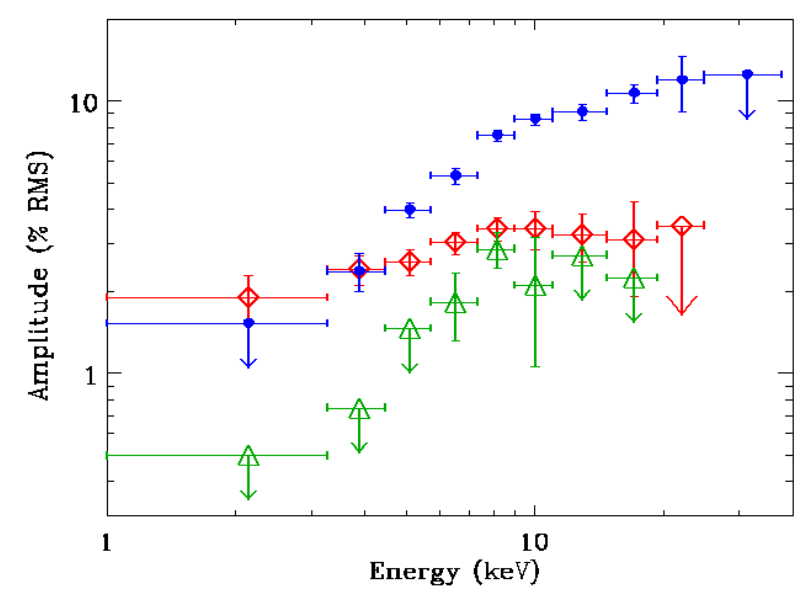

Figure 4: RMS spectra obtained during Obs. 1 for Peak 1 (red) Peak 2 (blue) and for the additional Lorentzian used to better represent Peak 2 (green).

It is pretty clear from Fig. 4 that the two main peaks show a quite different behaviour. Peak 2 has a steeper (harder) spectrum than Peak 1. The latter first increases up to $\sim 5.7 \mathrm{keV}$ and is then flat until $\sim 20 \mathrm{keV}$. Peak 2 is undetectable in the first energy bin (and is thus fainter than Peak 1). It increases up to $\sim 20 \mathrm{keV}$ where its plateau is reached.

\section{Conclusions}

While peaks 1 and 2 have frequencies that are in a harmonical relationship, they do not share the same properties. They have opposite signs of their time lags (Casella et al. 2004), their temporal evolution is clearly different (Sec. 3), and their spectra have different slopes and cut-off energies (Sec. 5). The first tempting conclusion one could draw out of these results is that the integer factor between the two frequencies is fortuitous and the peaks are not related. This interpretation is, however, difficult to reconcile with the fact that the same type of QPO is commonly seen with harmonics. In addition the two observations presented here are separated by an observation showing another type of QPO, which makes it difficult to believe that in both the same fortuitous phenomenon occurred.

The difference of spectral shape, in particular the fact that Peak 2 is harder, may indicate that the signal giving birth to the QPO is more sinusoidal at high energies (Rao et al. 2010, in the case of XTE J1550-564). This, however, does not account for the different signs of the time lags. In addition Rao et al. (2010) also mention that in fact the lowest frequency peak could be the fundamental. In that case the 'more sinusoidal' interpretation does not hold.

All this suggest that, although some common physics might set the frequencies of the peaks, 
the origin of the two QPO is distinct ${ }^{1}$. Their different dependence on the count rate may indicate a kind of competing mechanism. This could be the case, for example, if the two peaks represent different modes of the same physical mechanism, that would be favoured at different moments.

\section{References}

Brocksopp, C., Fender, R. P., McCollough, M., et al. 2002, MNRAS, 331, 765

Casella, P., Belloni, T., Homan, J., \& Stella, L. 2004, A\&A, 426, 587

Casella, P., Belloni, T., \& Stella, L. 2005, ApJ, 629, 403

Cui, W. 1999, ApJ, 524, L59

Cui, W., Shrader, C. R., Haswell, C. A., \& Hynes, R. I. 2000, ApJ, 535, L123

Homan, J. \& Belloni, T. 2005, Ap\&SS, 300, 107

Homan, J., Wijnands, R., van der Klis, M., et al. 2001, ApJS, 132, 377

Mikles, V. J., Varniere, P., Eikenberry, S. S., Rodriguez, J., \& Rothstein, D. 2009, ApJ, 694, L132

Muno, M. P., Morgan, E. H., \& Remillard, R. A. 1999, ApJ, 527, 321

Rao, F., Belloni, T., Stella, L., Zhang, S. N., \& Li, T. 2010, ApJ, 714, 1065

Remillard, R. A. \& McClintock, J. E. 2006, ARA\&A, 44, 49

Remillard, R. A., Sobczak, G. J., Muno, M. P., \& McClintock, J. E. 2002, ApJ, 564, 962

Rodriguez, J. \& Varnière, P. 2011, ApJ, accepted

Rodriguez, J., Corbel, S., Hannikainen, D. C., et al. 2004a, ApJ, 615, 416

Rodriguez, J., Corbel, S., Kalemci, E., Tomsick, J. A., \& Tagger, M. 2004b, ApJ, 612, 1018

Rodriguez, J., Durouchoux, P., Mirabel, I. F., et al. 2002a, A\&A, 386, 271

Rodriguez, J., Shaw, S. E., Hannikainen, D. C., et al. 2008, ApJ, 675, 1449

Rodriguez, J., Varnière, P., Tagger, M., \& Durouchoux, P. 2002b, A\&A, 387, 487

Shaposhnikov, N. \& Titarchuk, L. 2007, ApJ, 663, 445

Varnière, P., Tagger, M., \& Rodriguez, J. 2011, A\&A, 525, A87+

Vignarca, F., Migliari, S., Belloni, T., Psaltis, D., \& van der Klis, M. 2003, A\&A, 397, 729

Wood, A., Smith, D. A., Marshall, F. E., \& Swank, J. 1999, IAU Circ., 7274, 1

\footnotetext{
${ }^{1}$ We remark here that, contrary to what we first reported in Rodriguez \& Varnière (2011), the bicoherence shows possible coupling between the two peaks $\left(b^{2}(3,3)\right.$ is rather high). The low value we obtain at $(\sim 3, \sim 6)$ indicates low coupling in the triplet $(\sim 3, \sim 6, \sim 9)$ (Maccarone private comm.). This, however does not change the "competing" mode interpretation as the physics giving birth to the modes is common.
} 